\title{
Electron spectrum of intercalated stage ordered layered structures: Periodic Anderson model approach
}

\author{
Stasyuk I. V., Velychko O. V. \\ Institute for Condensed Matter Physics \\ of the National Academy of Sciences of Ukraine \\ 1 Svientsitskii Str., 79011, Lviv, Ukraine
}

(Received 2 December 2015)

\begin{abstract}
Influence of intercalation on the electronic band structure of the layered nanohybrid compound of the GaSe-type with a stage ordering (three layers in the packet in the considered case) is studied in the modified version of the periodic Anderson model. Density of electron states for the intercalated system is calculated both in the impurity single-level approximation and the one with the level smearing out (of the Lorentzian-type) due to local electron correlations. Intercalated particles form an additional band (usually placed near the bottom of the main band) like the narrow impurity band (being far enough from the main band) or the more extended band hybridized with the main one (for the case of overlapping). The most pronounced transformation of the main band takes place in the vicinity of the impurity level. Changes in the total density of electron states due to the broadening of impurity levels and the increase of the intercalant concentration are analyzed.
\end{abstract}

Keywords: layered crystals, stage ordering, intercalation, electronic spectrum, density of states

2000 MSC: 81 V99

UDC: $51-73 ; 538.915 ; 544.22 ; 546.22 / .24$

\section{Introduction}

Layered semiconductors are well known materials investigated for decades [1]. Gallium and indium selenides have aroused considerable interest because of their potential implementation in nonlinear optical devices, solar cells, high sensitive infrared, pressure and tensometric transducers and solid state power generators. These crystals have been proposed as possible photovoltaic and photoconductive materials, because their band gaps are relatively large and match the visible spectrum well. More recently, InSe has been considered as a possible cathode material for integrable solid state microbatteries. The remarkable feature for this purpose is the easy intercalation of lithium atoms in the space between layers without significant change in volume. When the intercalation occurs, the intercalant atoms enter the van der Waals gaps between the layers. Ba or Li intercalated indium selenide can be applied as solid solution electrodes, in particular in those cases where intercalation is possible over a wide stoichiometry range [2].

There exists a technology of a stage ordering of a host compound (monochalcogenides of indium and gallium in our case) aimed at a better penetration of intercalant particles into the matrix as well as a possibility of intercalation of large molecular groups like oligo-dimethylamino-methacrylate [3]. Such a stage ordering is perpendicular to the host layers with a significant increase of the distance between the packets (mostly three layers in the packet in the considered case [3]).

Layered crystals are characterized by an anisotropic dispersion law $\varepsilon(\boldsymbol{k})$ for the electron energy which is known as the Fivaz law [4,5]. Numerical calculations for specific compounds are performed in the framework of various methods: from a simple tight binding approximation based on the localized atomic orbitals (LCAO) to the sophisticated schemes utilizing the density functional theory (DFT) 
and various $a b$ initio techniques. Calculations performed for the semiconductor layered systems $[6,7]$ certify the reliability of the LCAO method which reproduces main features of the electron spectrum obtained with the use of the LMTO scheme (a version of the DFT in the local density approximation). A simple LCAO approach was successful in the calculation of the electron spectrum of two-, three(and more) layer graphene [8].

Energy gaps related to the electron motion perpendicularly to the layers can overlap with the continuous spectrum describing the electron motion within the layers. Such an effect was never studied for the case of stage ordering as well as no analysis of peculiarities of the electron spectrum was made for the systems with this kind of lattice modulation.

Our previous study [9] was devoted to calculation of the band electron spectrum of the stage ordered layered crystal prepared by the intercalation-deintercalation cycle with unoccupied widened gaps between the packets. We use the tight binding approximation based on the LCAO scheme considering nondegenerate electron orbitals localized on the atoms of separate layers as the basis ones. Besides the band spectrum, densities of electron states were calculated and their peculiarities at different relations between the main model parameters were studied. The step-like shape of density of states near the band edge is an important feature of the obtained spectrum. Such a feature is a generic property of layered superlattices and heterostructures [9].

In the present work we will investigate changes in electron spectrum and density of electron states in such a system which are caused by influence of particles (atoms, atomic groups or complex molecules) intercalated into the gaps between the packets. Our study will be performed in the framework of the above mentioned LCAO scheme with use of a model approach for description of the single-electron spectrum of separate intercalated particles. In this model approach a sophisticated structure of the density of impurity energy states is approximated by a smooth function of the Lorentzian type. It takes simultaneously into account the electron correlations (e.g. damping effects). Such an approach reveals cardinal peculiarities of electron spectrum like emergence of the impurity band, occurrence of the gap in the spectrum and rise of additional peaks and singularities caused by hybridization of the band and the localized states.

\section{Model Hamiltonian and equations of motion}

Let us introduce the model similar to the periodic Anderson model [11], to describe the electron excitations in the layered matrix which is intercalated by some particles (having, in general, a complex electron spectrum):

$$
\widehat{H}=\sum_{k} \varepsilon_{k} a_{k}^{+} a_{k}+\sum_{i}^{*} \sum_{p} \lambda_{p} X_{i}^{p p}+\frac{1}{\sqrt{N}} \sum_{i}^{*} \sum_{k}\left(V_{k} \mathrm{e}^{\mathrm{i} k R_{i}} a_{k}^{+} \xi_{i}+V_{k}^{*} \mathrm{e}^{-\mathrm{i} k R_{i}} \xi_{i}^{+} a_{k}\right)
$$

where the first term describes the band spectrum of the "bare" layered structure $\left(\varepsilon_{k}\right.$ is the energy of electron spectrum with wave vector $\boldsymbol{k}, \mu$ is the chemical potential, $a_{k}$ and $a_{k}^{+}$are annihilation and creation operators with respective wave vectors), the second term characterizes the structure of electron spectrum of the intercalated particle $\left(\lambda_{p}\right.$ is the energy of the respective state and $X_{i}^{p p}$ is the projection operator into this state for the $i$-th particle; the sum $\sum_{i}^{*}$ is limited to sites occupied by intercalated particles), and the third one describes the electron transfer from the band to an intercalated particle and vice versa (so called hybridization of band and localized states);

$$
\xi_{i}=\sum_{q q^{\prime}} A_{q q^{\prime}} X_{i}^{q q^{\prime}}, \quad \xi_{i}^{+}=\sum_{q q^{\prime}} A_{q q^{\prime}}^{*} X_{i}^{q^{\prime} q}=\sum_{q q^{\prime}} A_{q^{\prime} q}^{*} X_{i}^{q q^{\prime}}
$$

Mathematical Modeling and Computing, Vol. 2, No. 2, pp. 191-203 (2015) 
Here operators of annihilation $\xi_{i}$ and creation $\xi_{i}^{+}$of an electron on an intercalated particle are expressed via Fermi-type Hubbard operators ([12]; see also [13]). The following commutation relations

$$
\left[X_{i}^{q q^{\prime}}, X_{j}^{p p^{\prime}}\right]_{+}=\delta_{i j}\left(X_{i}^{q p^{\prime}} \delta_{q^{\prime} p}+X_{i}^{p q^{\prime}} \delta_{p^{\prime} q}\right), \quad\left[X_{i}^{q q^{\prime}}, X_{j}^{p p}\right]=\delta_{i j} X_{i}^{q q^{\prime}}\left(\delta_{q^{\prime} p}-\delta_{p q}\right)
$$

are fulfilled, where $\left[X_{i}^{q q^{\prime}}, X_{j}^{p p^{\prime}}\right]_{+}$is an anticommutator.

According to formulae (2) and (3), the equation of motion for the Hubbard operator $X_{i}^{q q^{\prime}}$ looks like

$$
\begin{aligned}
{\left[X_{i}^{q q^{\prime}}, \widehat{H}\right]=} & \left(\lambda_{q^{\prime}}-\lambda_{q}\right) X_{i}^{q q^{\prime}}+\frac{1}{\sqrt{N}} \sum_{j}^{*} \sum_{k} V_{k}^{*} \mathrm{e}^{-\mathrm{i} k R_{j}}\left[X_{i}^{q q^{\prime}}, \xi_{j}^{+}\right]_{+} a_{k}-\frac{1}{\sqrt{N}} \sum_{j}^{*} \sum_{k} V_{k} \mathrm{e}^{\mathrm{i} k R_{j}} a_{k}^{+}\left[X_{i}^{q q^{\prime}}, \xi_{j}\right]_{+} \\
= & \left(\lambda_{q^{\prime}}-\lambda_{q}\right) X_{i}^{q q^{\prime}}+\frac{1}{\sqrt{N}} \sum_{j}^{*} \sum_{k} V_{k}^{*} \mathrm{e}^{-\mathrm{i} k R_{j}}\left[X_{i}^{q q^{\prime}}, \sum_{p p^{\prime}} A_{p^{\prime} p}^{*} X_{j}^{p p^{\prime}}\right]_{+} a_{k} \\
& -\frac{1}{\sqrt{N}} \sum_{j}^{*} \sum_{k} V_{k} \mathrm{e}^{\mathrm{i} k R_{j}} a_{k}^{+}\left[X_{i}^{q q^{\prime}}, \sum_{p p^{\prime}} A_{p p^{\prime}} X_{j}^{p p^{\prime}}\right]_{+}
\end{aligned}
$$

Here

$$
\left[X_{i}^{q q^{\prime}}, \xi_{j}\right]_{+}=\delta_{i j}\left(\sum_{p^{\prime}} A_{q^{\prime} p^{\prime}} X_{i}^{q p^{\prime}}+\sum_{p} A_{p q} X_{i}^{p q^{\prime}}\right)
$$

and respectively

$$
\left[X_{i}^{q q^{\prime}}, \xi_{j}^{+}\right]=\delta_{i j}\left(\sum_{p^{\prime}} A_{p^{\prime} q^{\prime}}^{*} X_{i}^{q p^{\prime}}+\sum_{p} A_{q p}^{*} X_{i}^{p q^{\prime}}\right) .
$$

The number of electrons $N_{q}$ in the state $|q\rangle$ is by unity less comparing to the state $\left|q^{\prime}\right\rangle\left(N_{q}=N_{q^{\prime}}-\right.$ - 1) and the matrix element $A_{q^{\prime} p^{\prime}}$ is nonzero if $N_{q^{\prime}}=N_{p^{\prime}}-1$, so the operator $X_{i}^{p q^{\prime}}$ in the first term of expression (5) describes the transition $\left|q^{\prime}\right\rangle \rightarrow|p\rangle$ with a decrease of the electron number by two $\left(N_{q}=N_{p^{\prime}}-2\right)$.

A similar conclusion is applied to the second term of expression (5). By contrast, in formula (6) operators $X_{i}^{q p^{\prime}}$ and $X_{i}^{p q^{\prime}}$ describe transitions between states with equal numbers of electrons $\left(N_{q}=\right.$ $=N_{q^{\prime}}-1=N_{p^{\prime}}+1-1=N_{p^{\prime}}$ etc.).

After decoupling in equation of motion (4) in the spirit of the random phase approximation (when the $X$-operators are replaced by their average values) one can obtain

$$
\begin{aligned}
& {\left[X_{i}^{q q^{\prime}}, \xi_{j}^{+}\right]_{+} \rightarrow 0} \\
& {\left[X_{i}^{q q^{\prime}}, \xi_{j}^{+}\right]_{+} \rightarrow \delta_{i j}\left(\sum_{p^{\prime}} A_{p^{\prime} q^{\prime}}^{*}\left\langle X_{i}^{q q}\right\rangle \delta_{q p^{\prime}}+\sum_{p} A_{q p}^{*}\left\langle X_{i}^{p p}\right\rangle \delta_{p q^{\prime}}\right)=\delta_{i j} A_{q q^{\prime}}^{*}\left\langle X_{i}^{q q}+X_{i}^{q^{\prime} q^{\prime}}\right\rangle .}
\end{aligned}
$$

Here, contributions from diagonal $X$-operators only survive after averaging.

Thus, equation of motion (4) takes the form

$$
\left[X_{i}^{q q^{\prime}}, \hat{H}\right]=\left(\lambda_{q^{\prime}}-\lambda_{q}\right) X_{i}^{q q^{\prime}}+\frac{1}{\sqrt{N}} \sum_{k} V_{k}^{*} \mathrm{e}^{-\mathrm{i} k \boldsymbol{R}_{i}} A_{q q^{\prime}}^{*}\left\langle X^{q q}+X^{q^{\prime} q^{\prime}}\right\rangle a_{k}
$$


It is used in the equation for a two-time Green's function [14] in a frequency representation

$$
\hbar \omega\left\langle\left\langle X_{i}^{q q^{\prime}} \mid a_{k^{\prime}}^{+}\right\rangle\right\rangle_{\omega}=\left(\lambda_{q^{\prime}}-\lambda_{q}\right)\left\langle\left\langle X_{i}^{q q^{\prime}} \mid a_{k^{\prime}}^{+}\right\rangle\right\rangle_{\omega}+\frac{1}{\sqrt{N}} \sum_{k} V_{k}^{*} \mathrm{e}^{-\mathrm{i} \boldsymbol{k} \boldsymbol{R}_{i}} A_{q q^{\prime}}\left\langle X^{q q}+X^{q^{\prime} q^{\prime}}\right\rangle\left\langle\left\langle a_{k} \mid a_{k^{\prime}}^{+}\right\rangle\right\rangle_{\omega} .
$$

This results in the expression

$$
\left\langle\left\langle X_{i}^{q q^{\prime}} \mid a_{k^{\prime}}^{+}\right\rangle\right\rangle_{\omega}=\frac{1}{\sqrt{N}} \sum_{k} V_{k}^{*} \mathrm{e}^{-\mathrm{i} \boldsymbol{k} \boldsymbol{R}_{i}} A_{q q^{\prime}}^{*} \frac{\left\langle X^{q q}+X^{q^{\prime} q^{\prime}}\right\rangle}{\hbar \omega-\lambda_{q^{\prime}}+\lambda_{q}}\left\langle\left\langle a_{k} \mid a_{k^{\prime}}^{+}\right\rangle\right\rangle_{\omega}
$$

and thus

$$
\left\langle\left\langle\xi_{i} \mid a_{k^{\prime}}^{+}\right\rangle\right\rangle_{\omega}=\frac{1}{\sqrt{N}} \sum_{k} V_{k}^{*} \mathrm{e}^{-\mathrm{i} \boldsymbol{k} \boldsymbol{R}_{i}} g_{0}(\omega)\left\langle\left\langle a_{k} \mid a_{k^{\prime}}^{+}\right\rangle\right\rangle_{\omega}
$$

where

$$
g_{0}(\omega)=\sum_{q q^{\prime}} A_{q q^{\prime}} A_{q q^{\prime}}^{*} \frac{\left\langle X^{q q}+X^{q^{\prime} q^{\prime}}\right\rangle}{\hbar \omega-\lambda_{q^{\prime}}+\lambda_{q}}
$$

is a so-called locator function (i.e. the electron Green's function for the intercalated particle). Such a locator function describes the spectrum of interlevel transitions (determined by energy differences $\lambda_{q^{\prime}}-\lambda_{q}$ ) taking place at appearance (disappearance) of an additional electron on the particle.

The band electron Green's function $\left\langle\left\langle a_{k} \mid a_{k^{\prime}}^{+}\right\rangle\right\rangle_{\omega}$ can be derived from the mixed Green's function (11). Starting from the equation of motion

$$
\left[a_{k}, \hat{H}\right]=\varepsilon_{k} a_{k}+\frac{1}{\sqrt{N}} \sum_{i}^{*} V_{k} \mathrm{e}^{\mathrm{i} \boldsymbol{k} \boldsymbol{R}_{i}} \xi_{i}
$$

one can write a set of equations

$$
\left(\hbar \omega-\varepsilon_{k}\right)\left\langle\left\langle a_{k} \mid a_{k^{\prime}}^{+}\right\rangle\right\rangle_{\omega}=\frac{\hbar}{2 \pi} \delta_{k k^{\prime}}+\frac{1}{\sqrt{N}} \sum_{i}^{*} V_{k} \mathrm{e}^{\mathrm{i} \boldsymbol{k} \boldsymbol{R}_{i}} \sum_{k_{1}} V_{k_{1}}^{*} \mathrm{e}^{-\mathrm{i} \boldsymbol{k}_{1} \boldsymbol{R}_{i}} g_{0}(\omega)\left\langle\left\langle a_{k_{1}} \mid a_{k^{\prime}}^{+}\right\rangle\right\rangle_{\omega} .
$$

We consider the case when intercalated particles randomly occupy their positions in the widened van der Waals gap, residing in the certain sites $i$ with equal probability. Now we can perform summation over all sites $\left(\sum_{i}^{*} \rightarrow x \sum_{i}\right)$ introducing a weight factor $x$ with meaning of concentration of intercalated particles. Such a simplification corresponds to the virtual crystal approximation [15].

Taking into account that

$$
\frac{1}{N} \sum_{i}^{*} \mathrm{e}^{\mathrm{i} \boldsymbol{k} \boldsymbol{R}_{i}} \mathrm{e}^{-\mathrm{i} \boldsymbol{k}_{1} \boldsymbol{R}_{i}} \rightarrow \frac{x}{N} \sum_{i} \mathrm{e}^{\left(\boldsymbol{k}-\boldsymbol{k}_{1}\right) \boldsymbol{R}_{i}}=x \delta_{k k_{1}}
$$

the expression (13) results in

$$
\left\langle\left\langle a_{k} \mid a_{k^{\prime}}^{+}\right\rangle\right\rangle_{\omega}=\frac{\hbar}{2 \pi} \frac{\delta_{k k^{\prime}}}{\hbar \omega-\varepsilon_{k}-x\left|V_{k}\right|^{2} g_{0}(\omega)} .
$$

Poles of this function determine electron energy spectrum. It can be found from the following equation

$$
\hbar \omega=\varepsilon_{k}+x\left|V_{k}\right|^{2} g_{0}(\omega) .
$$

A more complete information about the electron spectrum (e.g. the partial weights of various bands) gives the density of energy states which can be written in explicit form using the expression for the locator function $g_{0}(\omega)$. In the next paragraph we will consider some examples. The single-site density 
of electron energy states is described by the following expression (see [13])

$$
\rho(\hbar \omega)=-\frac{2}{\hbar} \operatorname{Im} \frac{1}{N} \sum_{k}\left\langle\left\langle a_{k} \mid a_{k}^{+}\right\rangle\right\rangle_{\omega+\mathrm{i} \varepsilon} .
$$

Later on we will derive this function utilizing some model form of the locator function $g_{0}(\omega)$. Of course, the strict calculations should be based on the exact energy spectrum of the intercalated particle, in particular on its part, that is close to the main electron band of the crystal.

\section{Density of states for intercalated system}

In the general case after separation of real and imaginary parts of function (16) the Green's function of electrons looks like

$$
\left\langle\left\langle a_{k} \mid a_{k}^{+}\right\rangle\right\rangle_{\omega+\mathrm{i} \varepsilon}=\frac{1}{2 \pi} \frac{1}{\omega-\varepsilon_{k} / \hbar-\left|\tilde{V}_{k}\right|^{2} g_{0}^{\prime}(\omega) / \hbar-\mathrm{i}\left|\tilde{V}_{k}\right|^{2} g_{0}^{\prime \prime}(\omega) / \hbar+\mathrm{i} \varepsilon},
$$

where $\left|\tilde{V}_{k}\right|^{2}=x\left|V_{k}\right|^{2}$ and

$$
g_{0}(\omega+\mathrm{i} \varepsilon)=g_{0}^{\prime}(\omega)+\mathrm{i} g_{0}^{\prime \prime}(\omega)
$$

is the single-site Green's function (analytically continued to the complex plane $\omega$ ) for the intercalated particle.

There are two following cases.

1. In the frequency region, where $g_{0}^{\prime \prime}(\omega) \neq 0$, one has:

$$
\begin{aligned}
\left\langle\left\langle a_{k} \mid a_{k}^{+}\right\rangle\right\rangle_{\omega+\mathrm{i} \varepsilon} & =\frac{1}{2 \pi} \frac{1}{\omega-\varepsilon_{k} / \hbar-\left|\tilde{V}_{k}\right|^{2} g_{0}^{\prime}(\omega) / \hbar-\mathrm{i}\left|\tilde{V}_{k}\right|^{2} g_{0}^{\prime \prime}(\omega) / \hbar} \\
& =\frac{1}{2 \pi} \frac{\omega-\varepsilon_{k} / \hbar-\left|\tilde{V}_{k}\right|^{2} g_{0}^{\prime}(\omega) / \hbar+\mathrm{i}\left|\tilde{V}_{k}\right|^{2} g_{0}^{\prime \prime}(\omega) / \hbar}{\left[\omega-\varepsilon_{k} / \hbar-\left|\tilde{V}_{k}\right|^{2} g_{0}^{\prime}(\omega) / \hbar\right]^{2}+\left[\left|\tilde{V}_{k}\right|^{2} g_{0}^{\prime \prime}(\omega) / \hbar\right]^{2}} .
\end{aligned}
$$

The respective imaginary part of Green's function is the following:

$$
\operatorname{Im}\left\langle\left\langle a_{k} \mid a_{k}^{+}\right\rangle\right\rangle_{\omega+\mathrm{i} \varepsilon}=\frac{1}{2 \pi} \frac{\mathrm{i}\left|\tilde{V}_{k}\right|^{2} g_{0}^{\prime \prime}(\omega) / \hbar}{\left[\omega-\varepsilon_{k} / \hbar-\left|\tilde{V}_{k}\right|^{2} g_{0}^{\prime}(\omega) / \hbar\right]^{2}+\left[\left|\tilde{V}_{k}\right|^{2} g_{0}^{\prime \prime}(\omega) / \hbar\right]^{2}} .
$$

2. In the frequency region, where $g_{0}^{\prime \prime}(\omega)=0$, the following expressions are relevant:

$$
\begin{aligned}
\left\langle\left\langle a_{k} \mid a_{k}^{+}\right\rangle\right\rangle_{\omega+\mathrm{i} \varepsilon} & =\frac{1}{2 \pi} \frac{1}{\omega-\varepsilon_{k} / \hbar-\left|\tilde{V}_{k}\right|^{2} g_{0}^{\prime}(\omega) / \hbar+\mathrm{i} \varepsilon}, \\
\operatorname{Im}\left\langle\left\langle a_{k} \mid a_{k}^{+}\right\rangle\right\rangle_{\omega+\mathrm{i} \varepsilon} & =-\frac{1}{2} \delta\left[\omega-\varepsilon_{k} / \hbar-\left|\tilde{V}_{k}\right|^{2} g_{0}^{\prime}(\omega) / \hbar\right] .
\end{aligned}
$$

The choice of the model Green's function $g_{0}(\omega)$ is a nontrivial problem depending on the structure and the nature of intercalated particles. We will restrict ourselves in what follows to the two simplest cases.

\subsection{Intercalated particle with a single electron level}

In the single-level approximation we will retain only one term in the expression (12)

$$
g_{0}(\omega)=\frac{b}{\hbar \omega-\lambda_{0}} .
$$

Mathematical Modeling and Computing, Vol. 2, No. 2, pp. 191-203 (2015) 
In this case only two states on the intercalated particle with electron occupation $n_{i}=0$ and $n_{i}=1$ (with the neglect of spin) are taken into account and there exists a single transition between these states with energy $\lambda_{0}$. This approximation allows one to study the basic changes of the electronic properties of the system caused by intercalation. Expressions for main characteristics of the spectrum can be obtained in analytical form thus greatly simplifying their subsequent analysis and application.

In this case the full Green's function is expressed by

$$
\left\langle\left\langle a_{k} \mid a_{k}^{+}\right\rangle\right\rangle=\frac{\hbar}{2 \pi} \frac{1}{\hbar \omega-\varepsilon_{k}-\left|\tilde{V}_{k}\right|^{2} \frac{b}{\hbar \omega-\lambda_{0}}}=\frac{1}{2 \pi} \frac{\omega-\lambda_{0} / \hbar}{\left(\omega-\varepsilon_{k} / \hbar\right)\left(\omega-\lambda_{0} / \hbar\right)-\tilde{b}},
$$

what defines the energy spectrum with two branches

$$
\omega_{1,2}=\frac{\varepsilon_{k}+\lambda_{0}}{2 \hbar} \pm \sqrt{\left(\frac{\varepsilon_{k}-\lambda_{0}}{2 \hbar}\right)^{2}+\tilde{b}}, \quad \tilde{b}=\frac{\left|\tilde{V}_{k}\right|^{2} b}{\hbar^{2}} .
$$

It is convenient to rewrite the Green's function separating contributions from every branch

$$
\left\langle\left\langle a_{k} \mid a_{k}^{+}\right\rangle\right\rangle=\frac{1}{2 \pi}\left(\frac{\omega_{1}-\varepsilon_{k} / \hbar}{\omega_{1}-\omega_{2}} \cdot \frac{1}{\omega-\omega_{1}}+\frac{\omega_{2}-\varepsilon_{k} / \hbar}{\omega_{2}-\omega_{1}} \cdot \frac{1}{\omega-\omega_{2}}\right)=\frac{1}{2 \pi}\left(\frac{A_{1}}{\omega-\omega_{1}}+\frac{A_{2}}{\omega-\omega_{2}}\right),
$$

where

$$
A_{1,2}=\frac{1}{2} \pm \frac{\varepsilon_{k}-\lambda_{0}}{2 \sqrt{\left(\varepsilon_{k}-\lambda_{0}\right)^{2}+4 \hbar^{2} \tilde{b}}} .
$$

This form of the Green's function simplifies calculation of the density of electron states $\rho(\hbar \omega)=\frac{1}{\hbar} \rho(\omega)$ :

$$
\rho(\omega)=-\frac{2}{N} \sum_{k} \operatorname{Im}\left\langle\left\langle a_{k} \mid a_{k}^{+}\right\rangle\right\rangle_{\omega+\mathrm{i} \varepsilon}=\frac{1}{N} \sum_{k}\left[A_{1}(k) \delta\left(\omega-\omega_{1 k}\right)+A_{2}(k) \delta\left(\omega-\omega_{2 k}\right)\right] .
$$

Let us replace the summation over wave vector in the first Brillouin zone by the integration

$$
\frac{1}{N} \sum_{k} A_{\alpha}(k) \delta\left(\omega-\omega_{\alpha k}\right)=\int \mathrm{d} x \rho_{0}(y) A_{\alpha}(y) \delta\left(\omega-\omega_{\alpha}(y)\right)
$$

where $\alpha=1,2 ; \rho_{0}(y)$ is the "unperturbed" density of band states (the density of states for the initial unintercalated system with band energy $\left.\varepsilon_{k}\right), \rho_{0}(y)=\frac{1}{N} \sum_{k} \delta\left(y-\varepsilon_{k}\right)$;

$$
A_{1,2}(y)=\frac{1}{2} \pm \frac{y-\lambda_{0}}{2 \sqrt{\left(y-\lambda_{0}\right)^{2}+4 \hbar^{2} \tilde{b}}}, \quad \omega_{1,2}(y)=\frac{y+\lambda_{0}}{2 \hbar} \pm \frac{1}{2 \hbar} \sqrt{\left(y-\lambda_{0}\right)^{2}+4 \hbar^{2} \tilde{b}} .
$$

The delta function in expression (27) can be rewritten in standard way

$$
\delta(f(y))=\sum_{i}\left|f^{\prime}\left(y_{i}\right)\right|^{-1} \delta\left(y-y_{i}\right),
$$

where $y_{i}$ are roots of the equation $f(y)=0$. In our case

$$
f^{\prime}\left(y_{1,2}\right)=-\frac{1}{2 \hbar}\left(1 \pm \frac{y_{0}-\lambda_{0}}{2 \sqrt{\left(y_{0}-\lambda_{0}\right)^{2}+4 \hbar^{2} \tilde{b}}}\right), \quad y_{0}=\frac{\hbar^{2} \omega^{2}-\lambda_{0} \hbar \omega-\hbar^{2} \tilde{b}}{\hbar \omega-\lambda_{0}} .
$$


In this case, the formula (27) for the density of electron states for intercalated system can be written down as

$$
\rho(\omega)=\rho_{0}\left(y_{0}\right) \sum_{\alpha=1}^{2}\left|f_{\alpha}^{\prime}\left(y_{0}\right)\right|^{-1} A_{\alpha}\left(y_{0}\right), \quad y_{0} \equiv y_{0}(\omega) .
$$

For layered system with stage ordering (three layers in the packet), the analytic form for the "unperturbed" density of states $\rho_{0}(y)$ was calculated in work [9] (see Appendix A).

Results of calculations of spectrum $\varepsilon_{\alpha}(\boldsymbol{k})=\hbar \omega_{\alpha_{k}}$ and density of electron states $\rho(\hbar \omega)=\rho(\omega) / \hbar$ are presented in figures 1 and $2\left(\left|\tilde{V}_{k}\right|^{2}\right.$ is replaced by a constant thus neglecting the dependence on $\left.\boldsymbol{k}\right)$.

An additional impurity branch (more strictly, a set of three branches for the system with three-layer packets) appears as a consequence of intercalation (see figure 1). Depending on the value of $\lambda_{0}$, the impurity band can degenerate into the impurity level (being far from the main band) or widen and hybridize with the main one (when they are close enough or one another overlap). In the considered approximation the gap between the bands always exists.

Changes in the density of electron energy states (figure 2) due to intercalation illustrate reconstruction of the system even better. If the energy of impurity level is far from the main band and placed below its bottom, the impurity band is narrow. Its width is also controlled by the parameter $w$ proportional to concentration of intercalated particles (e.g. increase of concentration $x$ enlarges corre-
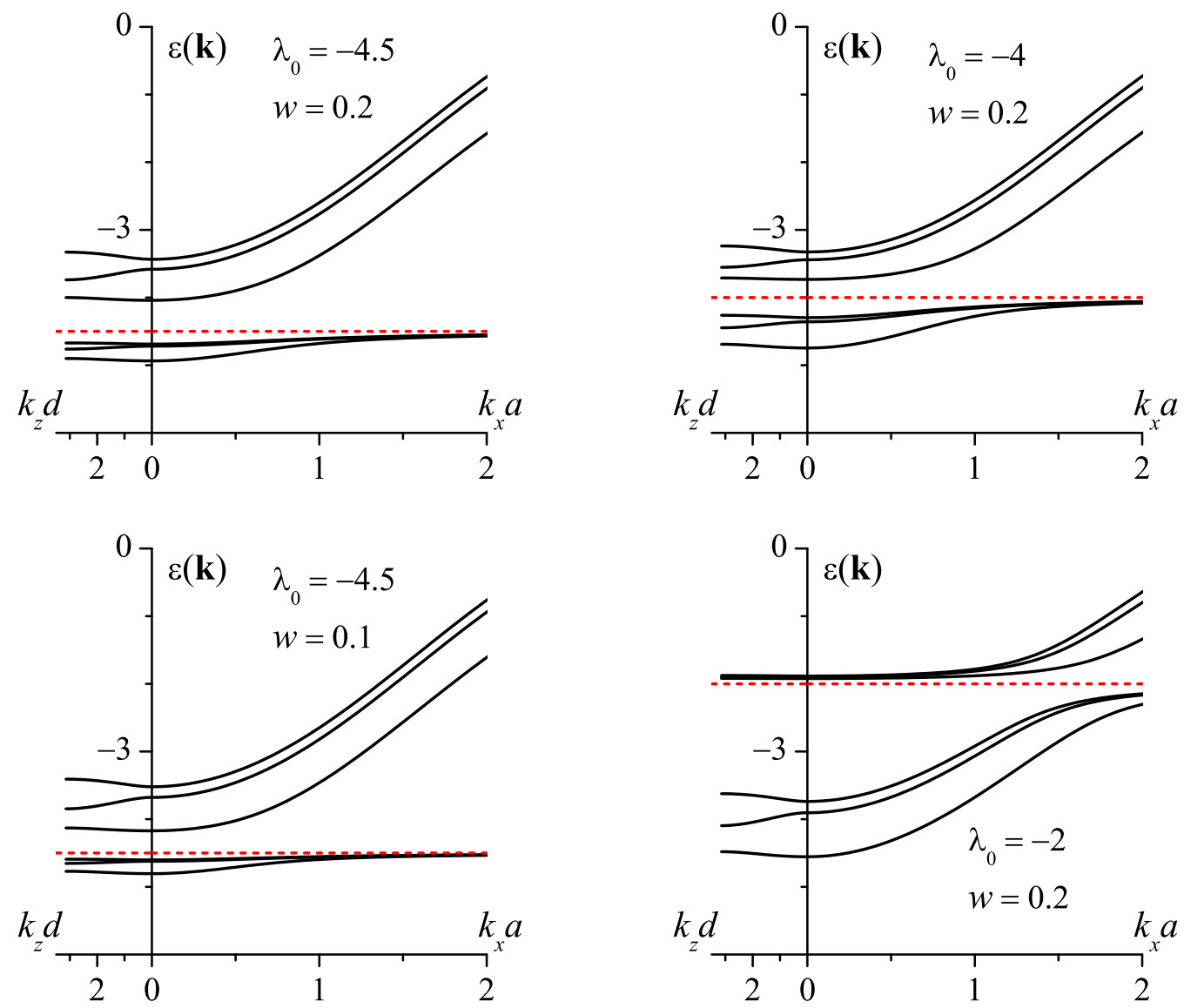

Fig. 1. The electron spectrum of the intercalated system. Influence of the localization $\left(\lambda_{0}\right)$ and the effective statistical weight $\left(w=x\left|V_{k}\right|^{2} b\right)$ of the intercalation induced band on the general structure of the spectrum. All model parameters with dimension of energy are made dimensionless using normalization by $t_{\perp}(t=0.3$, $\left.t^{\prime}=0.1, \delta=0.1\right)$. Here $t_{\perp}, t$, and $t^{\prime}$ are parameters of electron transfer in the layer, between the layers, and between the packets, respectively; $\delta=\varepsilon^{\prime}-\varepsilon$ is a difference between single-site electron energies in inner and outer layers of the packet (see [9]). Dashed lines mark the position of local energy level $\lambda_{0}$. 
spondingly the impurity peak). Hybridization of bands enhances when the impurity band approaches the main one. Only the bottom of the main band undergoes a significant reshaping while the rest of the band remains practically intact.
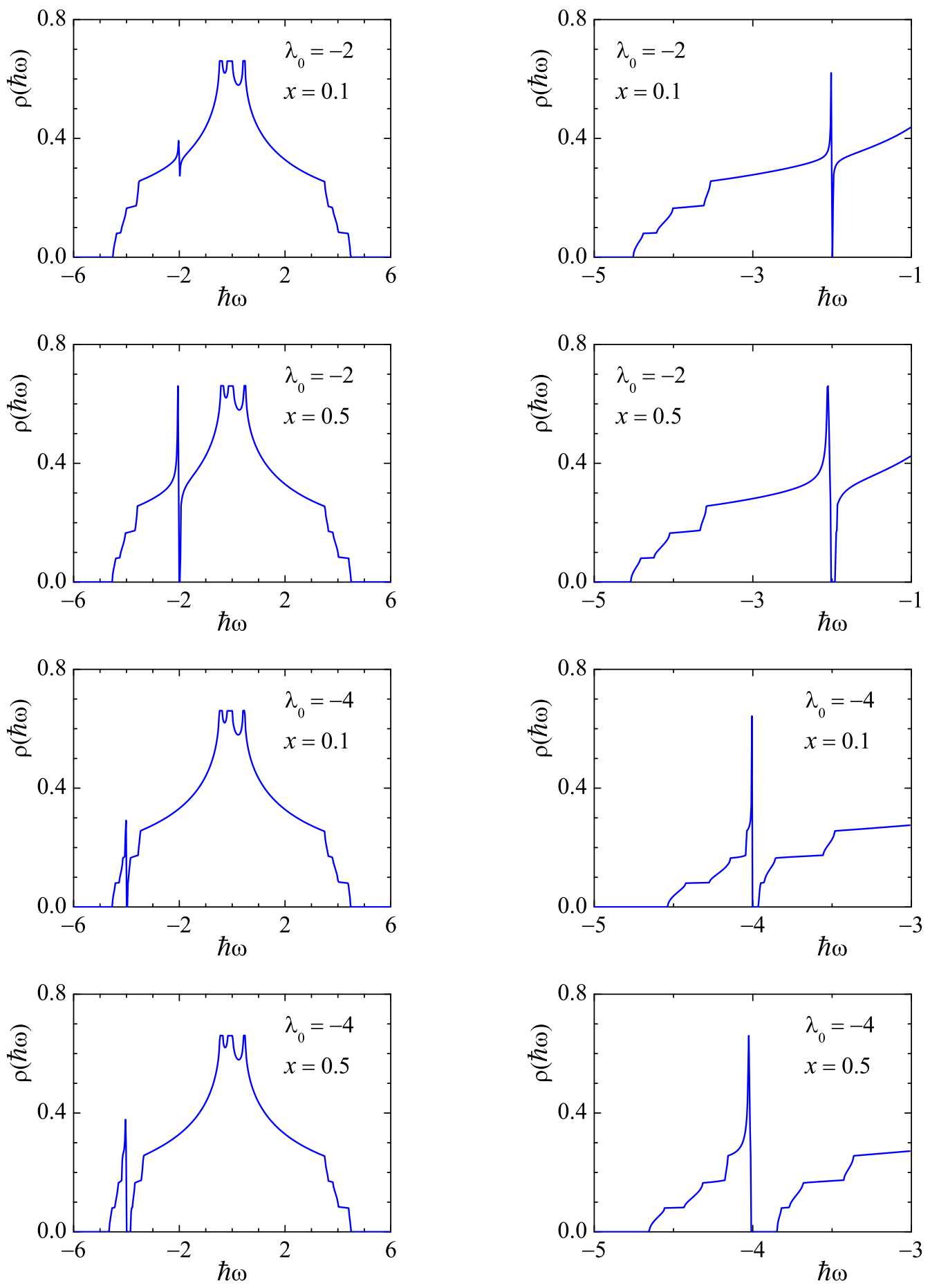

Fig. 2. Reconstruction of the density of electron energy states due to intercalation. The effect of localization $\left(\lambda_{0}\right)$ of impurity level at various intercalant concentrations $(x)$ is presented (the left column; the right one depicts enlarged view of the density of states in the region of the impurity level). Here $\delta=0.1$ and $\left|V_{k}\right|^{2} b=0.2 ; \Gamma=0$. All model parameters with dimension of energy are made dimensionless using normalization by $t_{\perp}(t=0.3$, $t^{\prime}=0.1, \delta=0.1$. 


\subsection{Widening of impurity electron level due to single-site electron interaction (scattering processes)}

The locator function $g_{0}$ is approximated in this case as

$$
g_{0}(\omega)=\frac{b}{\hbar \omega-\lambda_{0}+\mathrm{i} \Gamma} \equiv \frac{b / \hbar}{\omega-\lambda_{0} / \hbar+\mathrm{i} \gamma} .
$$

A nonzero imaginary part of the energy spectrum indicates a final lifetime of an electron on the level $\lambda_{0}$ (i.e. damping). One can separate the real and imaginary parts

$$
g_{0}^{\prime}(\omega)=\frac{b}{\hbar} \frac{1}{\omega-\lambda_{0} / \hbar}, \quad g_{0}^{\prime \prime}(\omega)=-\frac{b}{\hbar} \frac{\gamma}{\left(\omega-\lambda_{0} / \hbar\right)^{2}+\gamma^{2}} ;
$$

in this case $g_{0}^{\prime \prime} \neq 0$ for all frequencies.

Despite its simplicity the Lorentzian form of the imaginary part for the locator $g_{0}(\omega)$ fairly well models multilevel electron spectrum of complex structures (e.g. small clusters [16] or big molecules).

Now the imaginary part of the total electron Green's function for the whole system is

$$
\operatorname{Im}\left\langle\left\langle a_{k} \mid a_{k}^{+}\right\rangle\right\rangle_{\omega+\mathrm{i} \varepsilon}=-\frac{1}{2 \pi} \frac{\tilde{b} \gamma}{\left(\omega-\lambda_{0} / \hbar\right)^{2}+\gamma^{2}}\left\{\left[\omega-\varepsilon_{k} / \hbar-\frac{\tilde{b}}{\omega-\lambda_{0} / \hbar}\right]+\left[\tilde{b} \frac{\gamma}{\left(\omega-\lambda_{0} / \hbar\right)^{2}+\gamma^{2}}\right]^{2}\right\}^{-1} .
$$

Respectively, the total density of electron states (18), after transition from the summation over $\boldsymbol{k}$ to the integration with the "unperturbed" density of states $\rho_{0}$, is written down as

$$
\rho(\hbar \omega)=\frac{1}{\hbar} \rho(\omega)=\frac{2}{\pi} \int \mathrm{d} y \rho_{0}(y) \frac{b_{1} \Gamma}{\left(\hbar \omega-\lambda_{0}\right)^{2}+\Gamma^{2}}\left\{\left[\hbar \omega-y-\frac{b_{1}}{\hbar \omega-\lambda_{0}}\right]^{2}+\left[\frac{b_{1} \Gamma}{\left(\hbar \omega-\lambda_{0}\right)^{2}+\Gamma^{2}}\right]^{2}\right\}^{-1},
$$

where $b_{1}=\left|\tilde{V}_{k}\right|^{2} b \equiv x\left|V_{k}\right|^{2} b \equiv x w$.
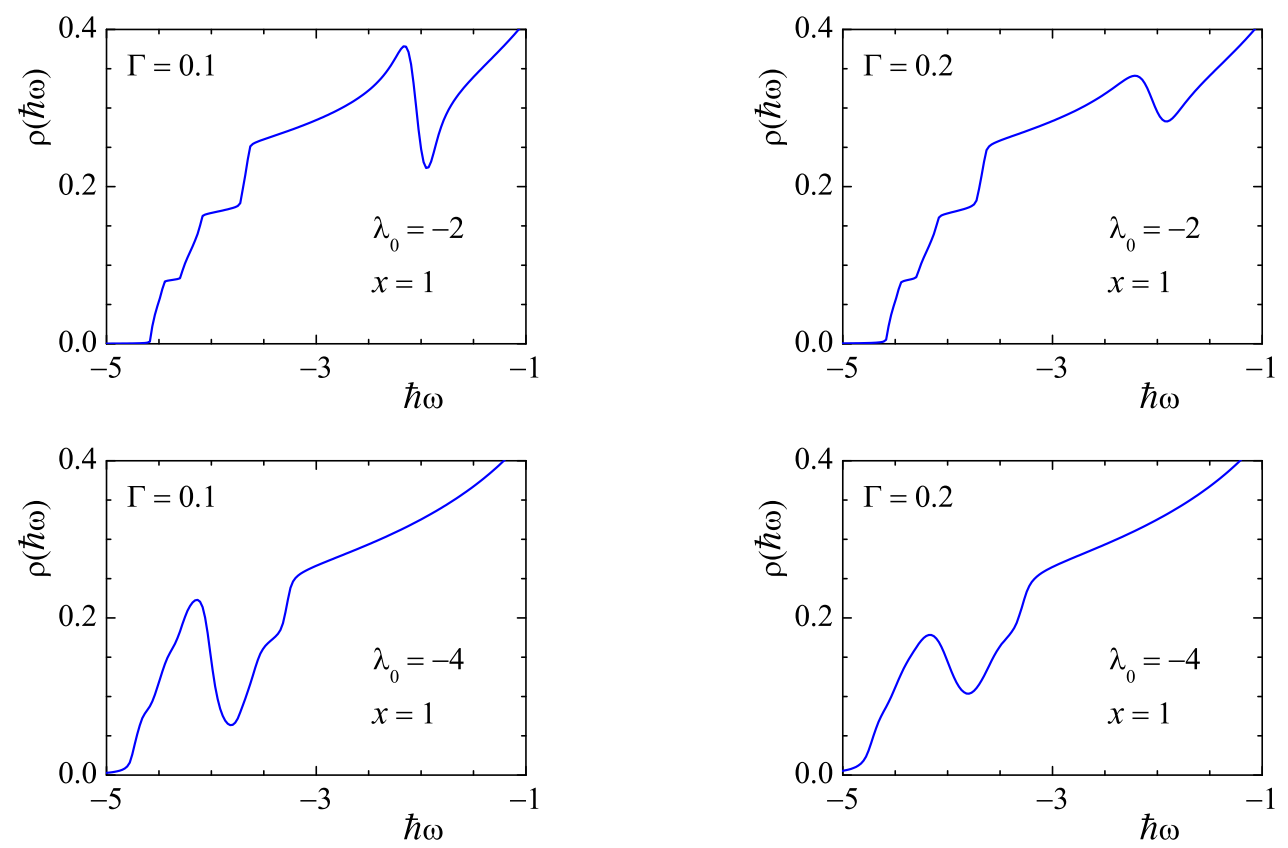

Fig. 3. The density of electron states for the intercalated system (enlarged view of the density of states in the region of the impurity level) at various values of damping $(\Gamma)$ of the impurity level and its localization $\left(\lambda_{0}\right)$; in all cases $x=1$. 

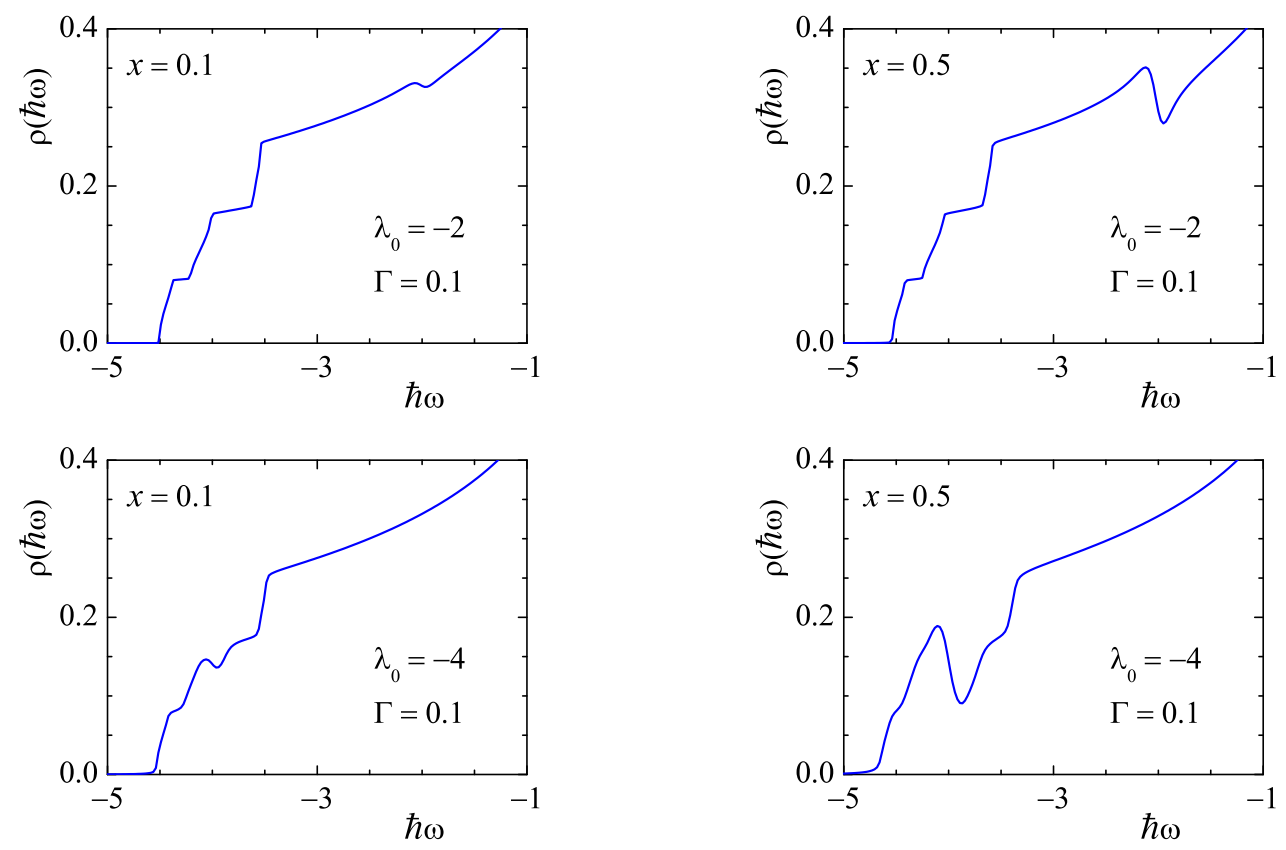

Fig. 4. The density of electron states for the intercalated system (enlarged view of the density of states in the region of the impurity level) at various values of concentration $(x)$ of the intercalant and localization $\left(\lambda_{0}\right)$ of the impurity level; in all cases $\Gamma=0.1$.

The total density of electron states for the intercalated system obtained (as previously) by numerical integration with the $\rho_{0}(y)$ function for the stage ordered layered crystal (three layers in the packet) is presented in figures 3 and 4 . One can see that increase of damping parameter $\Gamma$ leads to widening of the impurity band, smoothing of its edges, and, finally, disappearance of a gap between the bands. This effect takes place for the impurity level $\lambda_{0}$ below the bottom of the main band $(\lambda=-4.0)$ as well as for the overlapping impurity level $\left(\lambda_{0}=-2.0\right)$. In the first case a significant reconstruction of the main band near its bottom is observed (figure 3 ).

Profiles of the density of electron states $\rho(\hbar \omega)$ at various concentrations $x$ of intercalant are illustrated in figure 4 for the case of nonzero damping $(\Gamma)$ and respective widening of the initial level $\lambda_{0}$. Increase of the intercalant concentration $x$ leads primarily to appearance of the pseudogap in the spectrum and deepening of the respective local minimum of the density of states. The related peak of the function $\rho(\hbar \omega)$ is associated with the so called virtual impurity state (usually considered as the result of overlapping of the impurity band with the main one).

\section{Conclusions}

In the framework of a simple model of an intercalated layered system with the stage ordering, the changes of its electron spectrum due to intercalation are described. Calculations have been performed within the model which is a simplified version of the periodic Anderson model. Electron correlations forming structure of the single-impurity energy spectrum are taken into account by introduction of the effective locator Green's function. We have considered two cases: the single-level model and the single-level model with damping effects (which widen the level). The principal restructuring of the electron spectrum and the respective density of states consists in additional splitting, emergence of gap (or pseudogap) and appearance of the single impurity band besides the main one. Being far enough from the main band, the impurity band degenerates into the impurity level. Approaching each other they hybridize with reconstruction of the respective edge of the main band.

In the case, when the single-electron energy spectrum of the impurity particle is approximated by the density of states of the Lorentzian shape, the smearing out of the full spectral density takes 
place. At the increase of parameter $\Gamma$ (that characterizes the local level broadening degree), the singularities of the total density of states $\rho(\hbar \omega)$ gradually smear out, the hybridization gap in the energy spectrum disappears, and the three-step structure of density of states near the energy band edge becomes less distinctive. Similar changes in the $\rho(\hbar \omega)$ function take place at the increase of the intercalant concentration.

The applied approaches greatly simplify the internal energy structure of intercalated particles but they allow one to obtain expressions for the electron excitation spectrum and the density of states in analytic form. Obtained results provide a good qualitative description of the intercalation effect on the electron subsystem of intercalated stage ordered layered crystals.

\section{A. Density of electron states for stage ordered layered structure}

A determinant of the equation set for the respective Green's functions in the case of the stage ordered layered structure (three layers in the packet) looks like [9]

$$
D=\left(\xi^{2}-\delta^{2}\right)(\xi+\delta)-2 t^{2}(\xi+\delta)-\left(t^{\prime}\right)^{2}(\xi-\delta)-2 t^{2} t^{\prime} \cos \left(q_{z} d\right)
$$

where $\xi=\hbar \omega-t_{\perp} \gamma_{q}-\varepsilon_{0}, \gamma_{q}=\cos \left(q_{x} a\right)+\cos \left(q_{y} a\right)$. The determinant $D$ can be represented as a product

$$
D=\left(\xi-z_{1}\right)\left(\xi-z_{2}\right)\left(\xi-z_{3}\right),
$$

where $z_{\alpha}$ are roots of the equation $D(\xi)=0$.

The densities of electron states for outer (1 and 3) and inner (2) layers of the packet are

$$
\rho_{1}(\hbar \omega)=\rho_{3}(\hbar \omega)=\frac{1}{N} \sum_{q} \sum_{\alpha} A_{\alpha} \delta\left(\xi-z_{\alpha}\right), \quad \rho_{2}(\hbar \omega)=\frac{1}{N} \sum_{q} \sum_{\alpha} B_{\alpha} \delta\left(\xi-z_{\alpha}\right),
$$

where the following notations are used

$$
A_{\alpha}=\frac{z_{\alpha}^{2}-\delta^{2}-t^{2}}{\left(z_{\alpha}-z_{\beta}\right)\left(z_{\alpha}-z_{\gamma}\right)}, \quad B_{\alpha}=\frac{\left(z_{\alpha}+\delta\right)^{2}-\left(t^{\prime}\right)^{2}}{\left(z_{\alpha}-z_{\beta}\right)\left(z_{\alpha}-z_{\gamma}\right)}
$$

providing $\beta \neq \alpha, \gamma \neq \alpha$ ч $\beta \neq \gamma$. One can note that the roots $z_{\alpha}\left(q_{z}\right)$ depend on $q_{z}$ only, while $q_{x}$ and $q_{y}$ are present only in the $\delta$-function

$$
\delta\left(\xi-z_{\alpha}\right)=\delta\left[\hbar \omega-t_{\perp}\left(\cos q_{x} a+\cos q_{y} a\right)-\varepsilon_{0}-z_{\alpha}\right]
$$

Thus, the summation over $q_{z}$ can be performed independently from the summation over $q_{x}$ and $q_{y}$. After replacement of summation by integration over these variables, expressions (37) look like

$$
\begin{aligned}
& \rho_{1}(\hbar \omega)=\rho_{3}(\hbar \omega)=\frac{d}{2 \pi} \int_{-\pi / d}^{\pi / d} \mathrm{~d} q_{z} \sum_{\alpha} A_{\alpha}\left(q_{z}\right)\left\{\frac{a^{2}}{(2 \pi)^{2}} \iint_{-\pi / a}^{\pi / a} \mathrm{~d} q_{x} \mathrm{~d} q_{y} \delta\left[\hbar \omega-\varepsilon_{0}-t_{\perp} \gamma_{q}-z_{\alpha}\left(q_{z}\right)\right]\right\} \\
& \rho_{2}(\hbar \omega)=\frac{d}{2 \pi} \int_{-\pi / d}^{\pi / d} \mathrm{~d} q_{z} \sum_{\alpha} B_{\alpha}\left(q_{z}\right)\left\{\frac{a^{2}}{(2 \pi)^{2}} \iint_{-\pi / a}^{\pi / a} \mathrm{~d} q_{x} \mathrm{~d} q_{y} \delta\left[\hbar \omega-\varepsilon_{0}-t_{\perp} \gamma_{q}-z_{\alpha}\left(q_{z}\right)\right]\right\} .
\end{aligned}
$$

Let us introduce a "two-dimensional" density of electron states for for the layer lattice

$$
\rho_{\perp}(u)=\frac{a^{2}}{(2 \pi)^{2}} \iint_{-\pi / a}^{\pi / a} \mathrm{~d} q_{x} \mathrm{~d} q_{y} \delta\left(u-t_{\perp} \gamma_{q}\right) .
$$

In this case the expression in braces in (39) can be written as $\{\ldots\}=\rho_{\perp}\left[\hbar \omega-\varepsilon_{0}-z_{\alpha}\left(q_{z}\right)\right]$. 
Integration over $q_{z}$ is performed in a similar way utilizing a relation which is true for an arbitrary function $\Phi$ :

$$
\frac{d}{2 \pi} \int_{-\pi / d}^{\pi / d} \mathrm{~d} q_{z} \Phi\left(\cos q_{z} d\right)=\frac{1}{\pi} \int_{-1}^{1} \frac{\Phi(x) \mathrm{d} x}{\sqrt{1-x^{2}}} \equiv \int_{-1}^{1} \rho_{\|}(x) \Phi(x) \mathrm{d} x .
$$

It can be applied because the roots $z_{\alpha}$ depend on $\cos q_{z} d$ only. The function $\rho_{\|}(x)$ is a density of states of a one-dimensional lattice (i.e. a simple one-dimensional chain of atoms).

The above considerations result in the following expressions:

$$
\begin{aligned}
\rho_{1}(\hbar \omega)=\rho_{3}(\hbar \omega) & =\frac{1}{\pi} \int_{-1}^{1} \frac{\mathrm{d} x}{\sqrt{1-x^{2}}} \sum_{\alpha} A_{\alpha}(x) \rho_{\perp}\left[\hbar \omega-\varepsilon_{0}-z_{\alpha}(x)\right], \\
\rho_{2}(\hbar \omega) & =\frac{1}{\pi} \int_{-1}^{1} \frac{\mathrm{d} x}{\sqrt{1-x^{2}}} \sum_{\alpha} B_{\alpha}(x) \rho_{\perp}\left[\hbar \omega-\varepsilon_{0}-z_{\alpha}(x)\right] .
\end{aligned}
$$

Here, functions $z_{\alpha}(x)(\alpha=1,2,3)$ are obtained from the solutions $z_{\alpha}\left(q_{z}\right)$ of the equation $D=0$ replacing $\cos q_{z} d$ by $x$.

The integrals in formulae (42) can be interpreted as convolutions of densities of states of the onedimensional lattice (oriented perpendicularly to the layers) and the two-dimensional lattice (in the plane of the layers) with respective weights $A_{\alpha}(x)$ and $B_{\alpha}(x)$ for every layer in the packet. Thus, the full density of states related to the unit cell equals to

$$
\rho(\hbar \omega)=\sum_{n} \rho_{n}(\hbar \omega)
$$

Expressions (42) are general enough and are applicable to the lattices of the arbitrary periodic structure of layers (square, rectangular, hexagonal, etc.). Type of the lattice determines a particular formula for the "transverse" density of states $\rho_{\perp}$, which is expressed in the general case with the help of elliptical integrals of the first kind (see [17]).

[1] Mooser E., Schlüter M. The band-gap excitons in gallium selenide. Il Nuovo Cimento B 18, 164 (1973).

[2] Grygorchak I.I., Kovalyuk Z.D., Mintyanskii I.V. Photopolarization processes in $\mathrm{Li}_{x} \mathrm{GaSe}_{\mathrm{Se}} \mathrm{Li}_{x} \mathrm{InSe}$ intercalates. Solid State Physics 31, 222 (1989).

[3] Grygorchak I. I., Matulka D. V., Ivashchyshyn F. O., Zaichenko O. S., Mitina N. Ye., Moskvin M. M. Supramolecular assemblies of configuration inorganic semiconductor/oligomer. Physical surface engineering 10, 256 (2012).

[4] Fivaz R., Mooser E. Electron-phonon interaction in semiconducting layer structures. Phys. Rev. 136, A833 (1964).

[5] Fivaz R. Theory of layer structures. J. Phys. Chem. Solids. 28, 839 (1967).

[6] DiMasi E., Foran B., Aronson M.C., Lee S. Stability of charge-density waves under continuous variation of band filling in $\mathrm{LaTe}_{2-x} \mathrm{Sb}_{x}(0 \leqslant x \leqslant 1)$. Phys. Rev. B 54, 13587 (1996).

[7] Shin K.Y., Ru N., Fisher I.R., Condron C.L., Toney M.F., Wu Y.Q., Kramer M.J. Observation of two separate charge density wave transitions in $\mathrm{Gd}_{2} \mathrm{Te}_{5}$ via transmission electron microscopy and high-resolution X-ray diffraction. Journal of Alloys and Compounds 489, 332 (2010).

[8] Castro Neto A.H., Guinea F., Peres N.M.R., Novoselov K.S., Geim A.K. The electronic properties of graphene. Rev. Mod. Phys. 81, 109 (2009).

[9] Stasyuk I.V., Velychko O.V. The study of electronic states in highly anisotropic layered structures with stage ordering. J. Phys. Studies 18, 2002 (2014) [in Ukrainian].

[10] EsakiL. Advances in semiconductor superlattices, quantum wells and heterostructures. Journal de Physique Colloques 45(C5), C5-3 (1984).

Mathematical Modeling and Computing, Vol. 2, No. 2, pp. 191-203 (2015) 
[11] FuldeP. Electron correlations in molecules and solids. (Springer series in solid-state sciences; 100). Springer, Berlin Heidelberg (1995).

[12] Hubbard J. Electron correlations in narrow energy bands. IV. The atomic representation. Proc. R. Soc. Lond. A 285, 542 (1965).

[13] Stasyuk I.V. Green's functions in quantum statistics of solids. Lviv National University Publ., Lviv (2013) [in Ukrainian].

[14] Zubarev D.N. Double-time Green functions in statistical physics. Sov. Phys. Usp. 3, 320 (1960).

[15] Nordheim L. Zur Elektronentheorie der Metalle. I. Ann. Phys. 401, 607 (1931) [in German]; Nordheim L. Zur Elektronentheorie der Metalle. II. Ann. Phys. 401, 641 (1931) [in German]; Muto T. On the electronic structure of alloys. Scientific Papers of the Institute of Physical and Chemical Research (Tokyo) 34, 377 (1938).

[16] Ferrando R., Jellinek J., Johnston R.L. Nanoalloys: from theory to applications of alloy clusters and nanoparticles. Chemical Reviews, 108, 845 (2008).

[17] Pesz K., Munn R.W. Densities of states and anisotropy in tight-binding models. J. Phys. C: Solid State Phys. 19, 2499 (1986).

\title{
Електронний спектр інтеркальованих пакетно-впорядкованих шаруватих структур. Модель типу періодичної моделі Андерсона
}

\author{
Стасюк I. В., Величко О. В. \\ Інститут фізики конденсованих систем НАН Украӥни \\ вул. Свєнціцького, 1, 79011, Лъвів, Україна
}

\begin{abstract}
Вплив інтеркаляції на електронну зонну структуру шаруватої наногібридної сполуки типу GaSe зі стадійним впорядкуванням (три шари в пакеті в цьому випадку) вивчено у модифікованій версії періодичної моделі Андерсона. Густину електронних станів інтеркальованої системи розраховано як у наближенні однорівневої домішки, так і домішки з лоренцівським розмиттям рівня, спричиненим електронними кореляціями. Інтеркальовані частинки формують додаткову зону (зазвичай, розташовану біля дна основної зони) у вигляді вузької домішкової зони (за достатньої віддаленості від основної) або достатньо широкої, що гібридизується з основною (у разі їх перекриття). Найбільш виражена зміна основної зони відбувається поблизу домішкового рівня. Проаналізовано зміни у повній густині електронних станів, що виникають завдяки розширенню домішкових рівнів та збільшенню концентрації інтеркалянта.
\end{abstract}

Ключові слова: шаруваті кристали, стадійне впорядкування, інтеркалячія, електронний спектр, густина станів

2000 MSC: 81 V99

Удк: $51-73 ; 538.915 ; 544.22 ; 546.22 / .24$ 